\title{
Iron deposits in the red nucleus at long-term follow-up after hypertrophic olivary degeneration
}

\author{
Yangyingqiu Liu ${ }^{1}$, Bingbing Gao ${ }^{1}$, Yanwei Miao ${ }^{1}$, Xiang Dong $^{2}$ \\ ${ }^{1}$ Department of Radiology, First Affiliated Hospital of Dalian Medical University, Dalian, China; ${ }^{2}$ Department of Neurology, First Affiliated Hospital \\ of Dalian Medical University, Dalian, China
}

Correspondence to: Yanwei Miao. Department of Radiology, First Affiliated Hospital of Dalian Medical University, 222 Zhongshan Road, Xigang, Dalian, China. Email: ywmiao716@163.com.

Submitted Jul 05, 2020. Accepted for publication Nov 06, 2020.

doi: 10.21037/qims-20-832

View this article at: http://dx.doi.org/10.21037/qims-20-832

\section{Introduction}

Hypertrophic olivary degeneration (HOD) is an uncommon trans-synaptic degenerating disease characterized by hypertrophy of the inferior olivary nucleus (ION). The disease typically results from interruptions of connections in the dentato-rubro-olivary pathway (DROP) or GuillainMollaret triangle (GMT), which encompasses the ipsilateral red nucleus $(\mathrm{RN})$, ipsilateral ION, and contralateral dentate nucleus (DN). Before the advent of magnetic resonance imaging (MRI), HOD was only detectable by autopsy; however, MRI now makes it possible to diagnose HOD in vivo. Most reports about HOD are based on conventional MRI, with only one report using susceptibility-weighted imaging (SWI) (1), and no reports on combined application of SWI and diffusion tensor imaging (DTI). This report presents a case of unilateral HOD in a 66-year-old male 4 years post-cerebellar hemorrhage, who was examined with both SWI and DTI.

\section{Case report}

A 66-year-old man with a history of hypertension and type 2 diabetes mellitus presented with sudden-onset dizziness and vomiting. Computed tomography (CT) at admission on November 28, 2015, showed hemorrhage in the right cerebellar hemisphere (Figure 1). The patient's blood pressure was $180 / 110 \mathrm{mmHg}$ at this time, and he presented with ataxia on neurological examination. The patient was subsequently admitted to the Department of Neurology for medical treatment for 2 weeks. Six months later, the

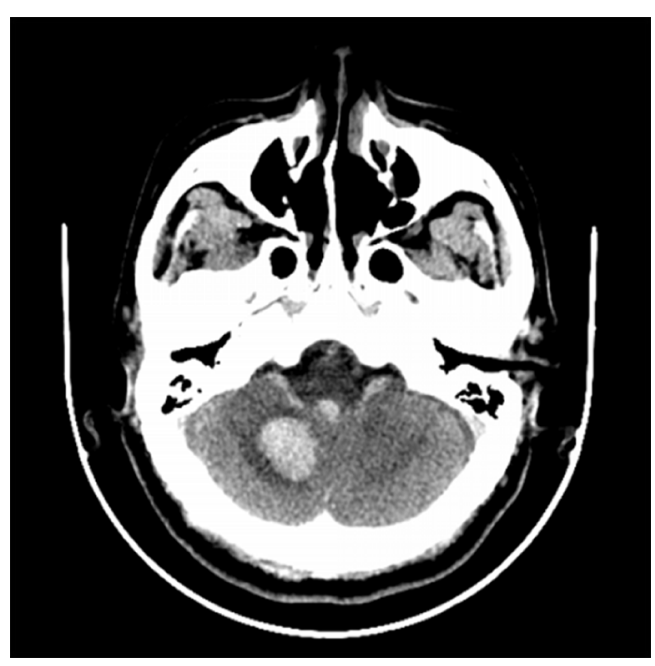

Figure 1 A CT scan showed a hemorrhage in the right cerebellar hemisphere.

patient was readmitted due to palatal tremor. On June 3, 2016, the first MRI scan revealed an oval lesion in the right cerebellum, T1-weighted image (T1WI) and T2-weighted image (T2WI) both showed hypointensity, which was consistent with chronic hemorrhage (Figure 2). A roundlike focus was also observed in the left ION, T2WI and diffusion-weighted imaging (DWI) showed hyperintensity. Eleven months after cerebellar hemorrhage, the patient developed slurred speech, vertigo, gait instability, and hypotonia of the right upper limb. Neurological examination revealed dysarthria, palatal tremor, and right dysmetria on finger-to-nose and heel-to-shin tests. On November 9, 

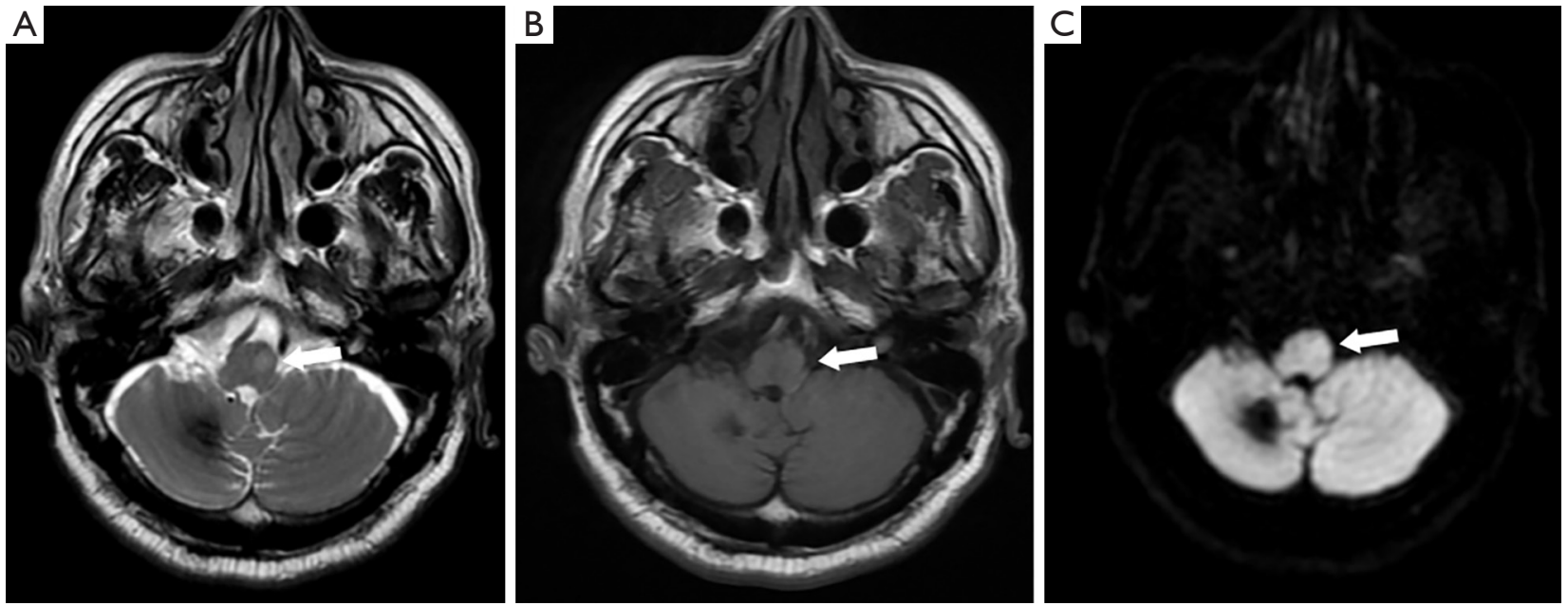

Figure 2 MRI at 6 months post-cerebellar hemorrhage. T2WI and T1WI (A,B) showed hypointensity in the right cerebellum, representing chronic hemorrhage; T2WI showed hyperintensity in the left ION without hypertrophy (white arrow); DWI (C) showed increased signal (white arrow). ION, inferior olivary nucleus.

2016, MRI showed enlargement of the left ION with T2WI hyperintensity and DWI isointensity without gadolinium enhancement (Figure 3), and SWI showed hypointensity in the right DN. HOD was diagnosed according to typical clinical and neuroimaging findings. Two years later, the patient developed deafness in the right ear and hearing loss in the left ear, with MRI showing gradual ION enlargement with persistent T2WI hyperintensity but isointensity on DWI. Four years later, the left ear hearing loss had increased in severity. On December 5, 2019, MRI showed ION hypertrophy, with persisting T2WI hyperintensity and DWI isointensity, and new right cerebellar atrophy (Figure 4). DTI showed reduced fractional anisotropy (FA) value in the left medulla oblongata compared to the contralateral side (left: 0.27 ; right: 0.30 ), and fiber tractography (FT) revealed reduced volume of the left central tegmental tract (CTT). SWI showed hypointensity in the right DN and showed reduced signal intensity in the left $\mathrm{RN}$ relative to the contralateral side, with an associated decreased phase value (left: -1.14 rad; right: $-0.41 \mathrm{rad}$ ).

\section{Discussion}

Various etiologies of HOD have been described, including cerebrovascular disease, tumor, trauma, surgery, and toxicity. In this case, HOD developed from a hemorrhage in the right $\mathrm{DN}$. The special trans-synaptic degeneration, which initially presents as hypertrophy rather than atrophy of the ION, is histopathologically characterized by enlarged and vacuolated neurons, astrocytosis, gliosis, and demyelination (2).

Anatomical knowledge of the Guillain-Mollaret triangle (Figure 5) is important for understanding the disease process, which occurs through four potential mechanisms: (I) lesions in the RN that lead to ipsilateral HOD, through ipsilateral CTT (rubro-olivary pathway), descending from the RN to the ipsilateral ION; (II) lesions in the $\mathrm{DN}$ and superior cerebellar peduncle (SCP) which lead to contralateral HOD, through ipsilateral dentato-rubro tract and the SCP (dentatorubro pathway) decussating within the midbrain to enter the contralateral RN; (III) lesions that disrupt the afferent nerve fiber input for both the CTT and the dentato-rubro tract, or lesions that are restricted to the intersection of the SCPT, which lead to bilateral HOD; (IV) lesions in the ION which cross the midline superiorly through the inferior cerebellar peduncle (ICP) which lead to contralateral cerebellar atrophy, through disruption to the contralateral DN (olivary-dentato pathway). In this case study, hemorrhage in the right DN resulted in hypertrophy of left ION, through the dentatorubro pathway. Peters et al. (3) also observed cerebellar atrophy in a patient with HOD and suggested that climbing fibers that synapse with Purkinje cells originate from the ION, and proposed trans-synaptic degeneration as the most plausible explanation for cerebellar atrophy.

In this case study, the phase values of bilateral RNs were lower than healthy elderly people $(-0.15 \pm 0.02 \mathrm{rad})$, which reported by Wang et al. (4) in a study of 14 healthy volunteers (mean age $64.3 \pm 12.7$ years; range $41-85$ years). This suggests that iron or other paramagnetic material 

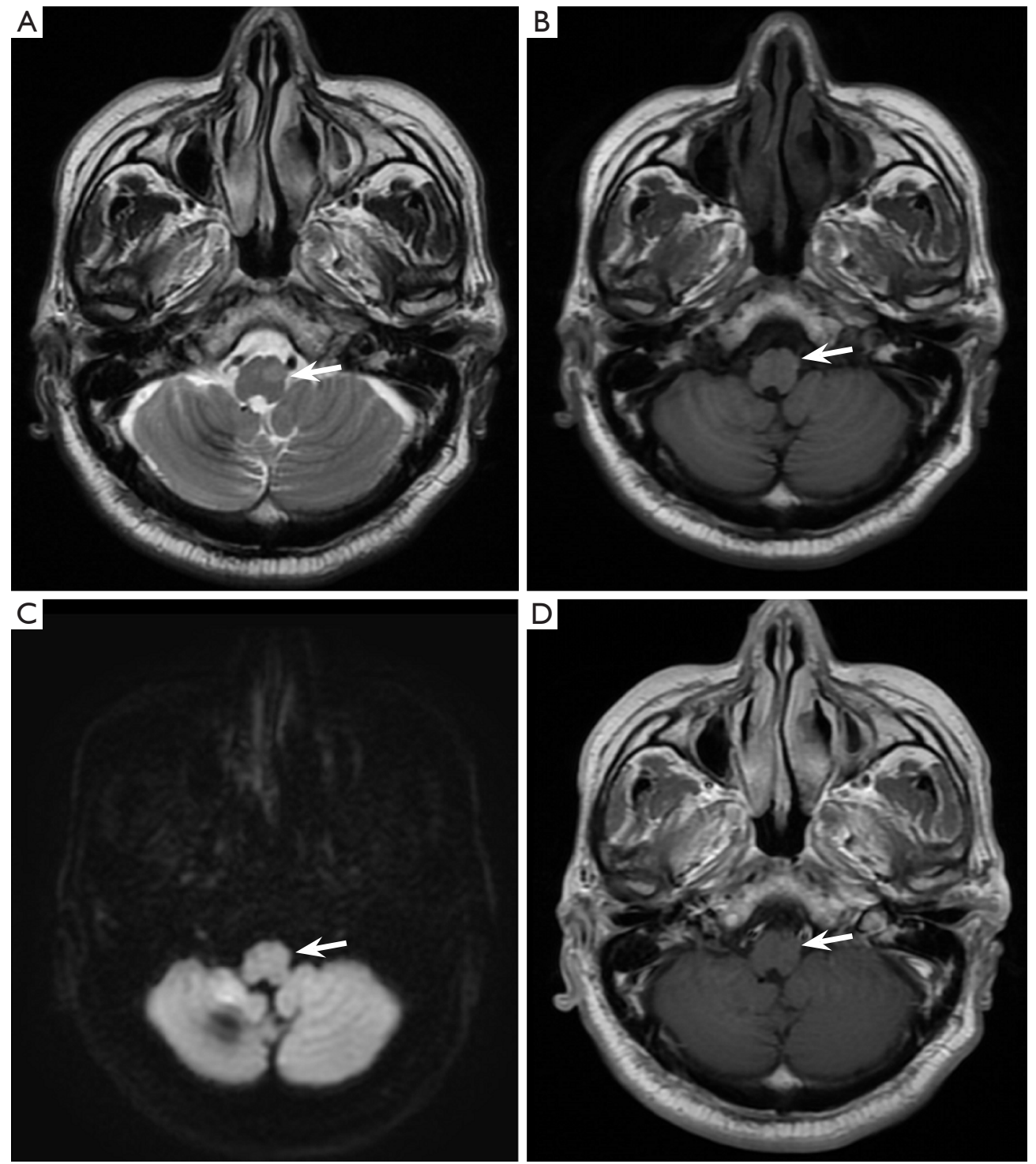

Figure 3 MRI at 11 months post-cerebellar hemorrhage. T2WI (A) showed enlargement of the ION and hyperintensity (white arrow); the lesion on T1WI (B) and DWI (C) demonstrated isointensity (white arrow); the lesion was non-contrast enhancement on contrast enhancement T1 (D) (white arrow). ION, inferior olivary nucleus.

deposition bilateral RNs were increased, and the deposition on the left side was more obvious. We speculated that the hemorrhagic lesion in the right $\mathrm{DN}$ caused microcirculation reduction, microglia aggregation, and further iron deposition in the left $\mathrm{RN}$ due to axonal degeneration in the dentato-rubro pathway; however, this hypothesis requires further confirmation.

Goyal et al. (5) described three distinct phases seen on MRI corresponding to the pathological changes of HOD. The first stage shows increased T2 signal in the
ION without hypertrophy, which usually occurs within 6 months of ictus. The second stage shows increased T2 signal and hypertrophy of the ION and is usually resolved within 3-4 years. During the third stage, olivary shrinkage becomes apparent on MRI, although increased T2 signal can persist. In previous studies (6), DWI was considered to show no restricted diffusion; however, in this case, DWI hyperintensity was observed in the left ION 6 months after cerebellar hemorrhage, but isointensity was observed at 11 months, 2 years, and 4 years, suggesting that signals on 

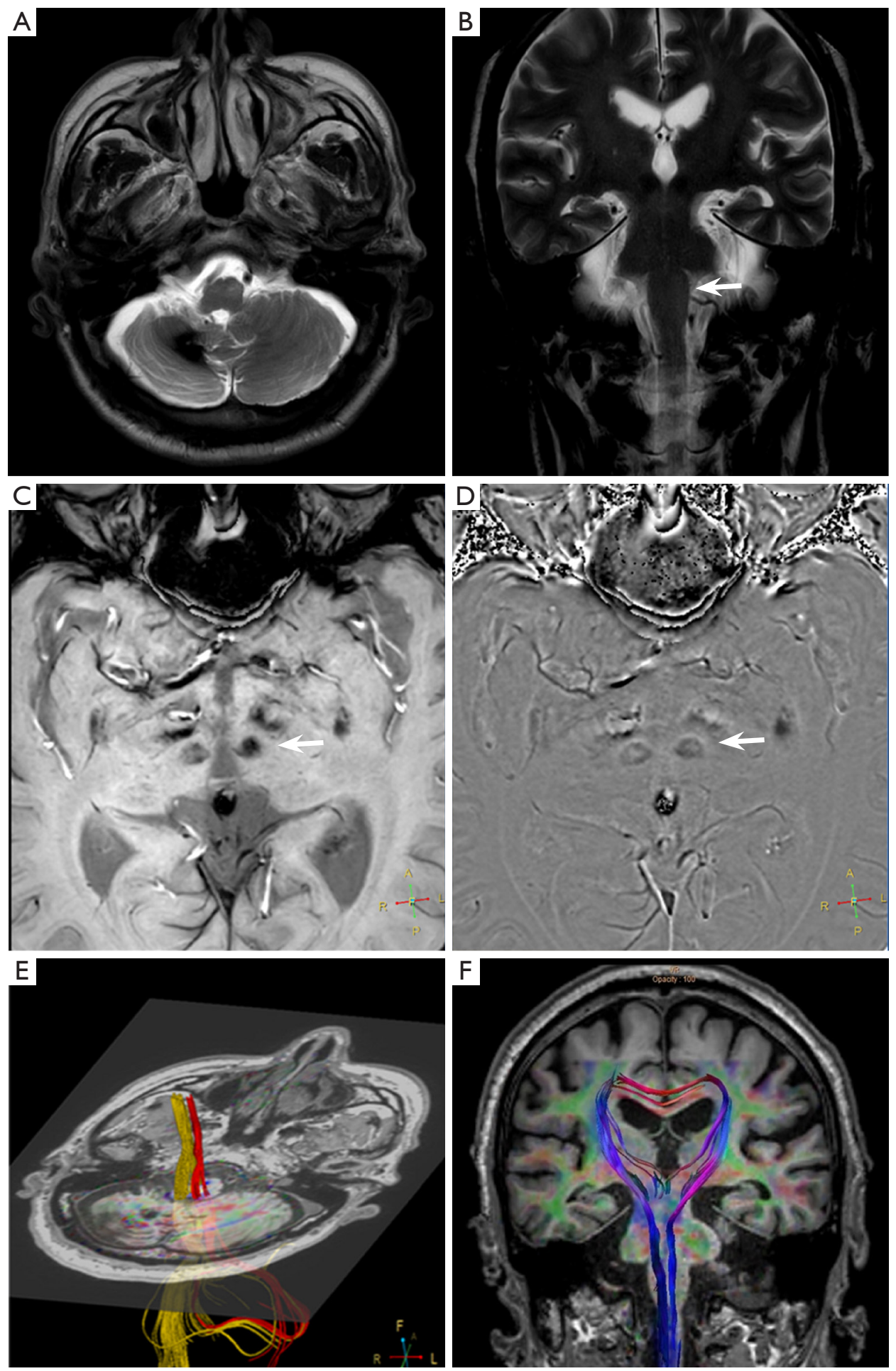

Figure 4 MRI 4 years post-cerebellar hemorrhage. Axial and coronal T2WI (A,B) showed enlargement and hyperintensity in the left ION persisted (white arrow), and right cerebellar atrophy. Susceptibility map (C) and phase map (D) showed that the iron deposition in the left RN (white arrow) had increased compared with the right; FT maps (E,F) showed the volume of the left CTT (red fibers in Figure 4E) at the level of the ION had decreased compared with the right CTT (yellow fibers in Figure 4E). ION, inferior olivary nucleus; RN, red nucleus; FT, fiber tractography; CTT, central tegmental tract. 


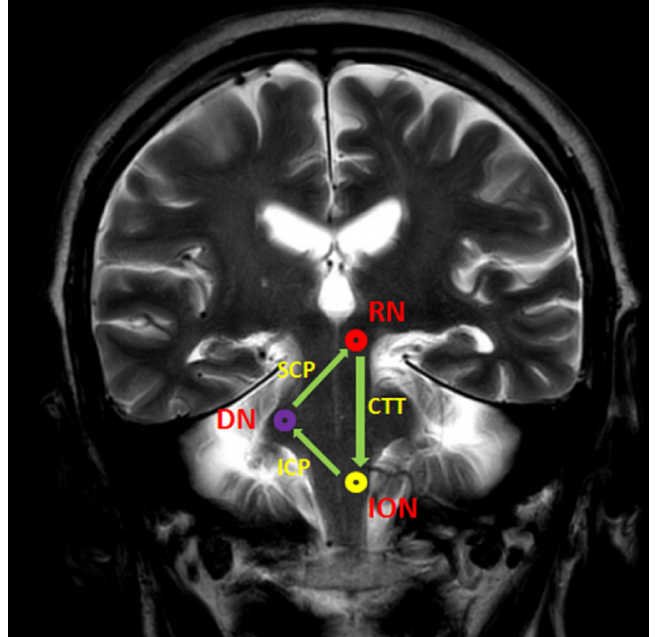

Figure 5 The Guillain-Mollaret triangle. RN, red nucleus; SCP, superior cerebellar peduncle; CTT, central tegmental tract; DN, dentate nucleus; ICP, inferior cerebellar peduncle; ION, inferior olivary nucleus.

DWI vary according to different periods of the lesion.

DTI allows the in vivo investigation of white matter fibers, which assists in analyzing and quantifying changes in DROP white matter fiber tracts in HOD patients. In this case study, the volume of the left CTT was decreased, which was consistent with previous studies (7). Moreover, FA value in the left ION was decreased, which might have been associated with demyelination.

In conclusion, SWI revealed the hypointensity of $\mathrm{RN}$, and DTI showed decreased volume of CTT in this patient, suggesting that SWI and DTI could be valuable methods for understanding the complicated changes in HOD.

\section{Acknowledgments}

Funding: This work was supported by The National Natural Science Foundation of China (No. 81671646).

\section{Footnote}

Conflicts of Interest: All authors have completed the ICMJE uniform disclosure form (available at http://dx.doi. org/10.21037/qims-20-832). The authors have no conflicts of interest to declare.

Ethical Statement: Written informed consent was obtained from the patient for publication of this manuscript and any accompanying images.

Open Access Statement: This is an Open Access article distributed in accordance with the Creative Commons Attribution-NonCommercial-NoDerivs 4.0 International License (CC BY-NC-ND 4.0), which permits the noncommercial replication and distribution of the article with the strict proviso that no changes or edits are made and the original work is properly cited (including links to both the formal publication through the relevant DOI and the license). See: https://creativecommons.org/licenses/by-nc-nd/4.0/.

\section{References}

1. Vossough A, Ziai P, Chatzkel JA. Red nucleus degeneration in hypertrophic olivary degeneration after pediatric posterior fossa tumor resection: use of susceptibilityweighted imaging (SWI). Pediatr Radiol 2012;42:481-5.

2. Tilikete C, Desestret V. Hypertrophic Olivary Degeneration and Palatal or Oculopalatal Tremor. Front Neurol 2017;8:302.

3. Peters J, Ding C, Amukotuwa S, Kempster P. Cerebellar atrophy with Chiari malformation: An example of transsynaptic degeneration? J Clin Neurosci 2019;69:279-80.

4. Wang C, Fan G, Xu K, Wang S. Quantitative assessment of iron deposition in the midbrain using 3D-enhanced T2 star weighted angiography (ESWAN): a preliminary crosssectional study of 20 Parkinson's disease patients. Magn Reson Imaging 2013;31:1068-73.

5. Goyal M, Versnick E, Tuite P, Cyr JS, Kucharczyk W, Montanera W, Willinsky R, Mikulis D. Hypertrophic olivary degeneration: metaanalysis of the temporal evolution of MR findings. AJNR Am J Neuroradiol 2000;21:1073-7.

6. Sobieraj P, Duczkowski M, Terczyńska I, Duczkowska A, Krupa K, Krupska E, Bekiesińska-Figatowska M. Hypertrophic olivary degeneration in a 16-year-old girl after subtotal surgery of a brainstem pilocytic astrocytoma - a case report. Pol J Radiol 2019;84:e68-e72.

7. Lai Q, Zheng C, Zhang R, Liu X, Li Y, Xu Q. MRI and Tractography in Hypertrophic Olivary Degeneration. Can J Neurol Sci 2018;45:332-3.

Cite this article as: Liu Y, Gao B, Miao Y, Dong X. Iron deposits in the red nucleus at long-term follow-up after hypertrophic olivary degeneration. Quant Imaging Med Surg 2021;11(7):3371-3375. doi: 10.21037/qims-20-832 